University of Windsor

Scholarship at UWindsor

1986

\title{
Lamb shifts and fine-structure splittings for light muonic ions: Hyperfine-structure corrections
}

\author{
R. A. Swainson \\ Gordon W. F. Drake \\ University of Windsor
}

Follow this and additional works at: https://scholar.uwindsor.ca/physicspub

Part of the Physics Commons

\section{Recommended Citation}

Swainson, R. A. and Drake, Gordon W. F.. (1986). Lamb shifts and fine-structure splittings for light muonic ions: Hyperfine-structure corrections. Physical Review A, 34 (1), 620-623.

https://scholar.uwindsor.ca/physicspub/89

This Article is brought to you for free and open access by the Department of Physics at Scholarship at UWindsor. It has been accepted for inclusion in Physics Publications by an authorized administrator of Scholarship at UWindsor. For more information, please contact scholarship@uwindsor.ca. 


\title{
Lamb shifts and fine-structure splittings for light muonic ions: Hyperfine-structure corrections
}

\author{
Robin Swainson and G. W. F. Drake \\ Department of Physics, University of Windsor, Windsor, Ontario, Canada N9B $3 P 4$
}

(Received 18 February 1986)

\begin{abstract}
Our previous calculations for the energy splittings of the states $2 s_{1 / 2}-2 p_{1 / 2}$ and $2 s_{1 / 2}-2 p_{3 / 2}$ for the muonic ions $\mu^{-}-\mathrm{Li}, \mu^{-}-\mathrm{Be}$, and $\mu^{-}-\mathrm{B}$ are extended to include hyperfine-structure corrections. The results show that there is a rich spectrum of well-resolved hyperfine transitions lying throughout the visible and infrared parts of the spectrum. A measurement of the transition frequencies would provide a precise determination of the nuclear radius and the hyperfine-structure coupling constants.
\end{abstract}

\section{INTRODUCTION}

Recent high-precision measurements of the $2 s_{1 / 2}-2 p_{3 / 2}$ transition frequencies in $\mu^{-}-\mathrm{He}$ (Refs. 1 and 2) have stimulated extensive calculations of the various quantum electrodynamic and finite-nuclear-size contributions to the energy levels. $^{3-5}$ Our previous work ${ }^{5}$ (henceforth referred to as I) showed that there exists a severe cancellation between the first-order vacuum polarization and finitenuclear-size contributions to the $2 s_{1 / 2}$ energy levels in the muonic systems $\mu^{-}-\mathrm{Li}, \mu^{-}-\mathrm{B}$, and $\mu^{-}-\mathrm{Be}$. (This phenomenon was previously reported in the estimates of Feinberg and Chen ${ }^{6}$ and Moskalev ${ }^{7}$ in connection with the possibility of detecting effects due to the weak interaction in muonic atoms.) The cancellation causes the $2 s_{1 / 2^{-}}$ $2 p_{1 / 2}$ and $2 s_{1 / 2}-2 p_{3 / 2}$ transition frequencies to fall in the visible or infrared regions of the spectrum, thus making them accessible to tunable laser sources. Hence, relatively low-precision measurements of these transition wavelengths would provide a very sensitive probe of nuclear size and structure.

In I, the effects of the hyperfine interactions on the quantum electrodynamic fine-structure levels were not considered. The hyperfine interaction energy nominally scales as $Z^{3}$. Combined with the fortuitous cancellation of the vacuum polarization and finite-nuclear-size corrections to the energy levels, this scaling gives rise to proportionately much larger hyperfine splittings than what one normally expects. Furthermore, while the nonrelativistic transition energies scale as only the first power of the reduced mass, $\mu$, of the system, the hyperfine interaction scales as $\mu^{3}$. Thus hyperfine interactions are considerably more important in muonic systems than in the corresponding electronic systems.

In this note we present calculations of the effect of hyperfine interactions on the wavelengths of the $2 s_{1 / 2}-2 p_{1 / 2}$ and $2 s_{1 / 2}-2 p_{3 / 2}$ transitions in the muonic systems $\mu^{-}-\mathrm{Li}$, $\mu^{-}-\mathrm{B}$, and $\mu^{-}-\mathrm{Be}$ obtained in $\mathrm{I}$, thus extending that work to the degree of precision required for accurate nuclear radius measurements. We also calculated the transition wavelengths in $\mu^{-}$-He for comparison with the previously published values of Borie. ${ }^{4}$

A further motivation for studying a heavier muonic system such as $\mu^{-}-\mathrm{Li}$ is that it may shed some additional light on the anomalously low collisional quenching rate observed at high pressures with $\mu^{-}-\mathrm{He}^{1,2}$ These measurements are in conflict with low-pressure quench-rate measurements $^{8}$ and with calculations. ${ }^{9,10}$ Attempts to explain the low quench rates at high pressures by means of a cluster surrounding the muonic ion ${ }^{11}$ have recently been criticized. $^{12}$

In Sec. II we briefly discuss the theoretical basis of our hyperfine interaction calculations, and in Sec. III we present the results of those calculations.

\section{THEORY}

The quantum-electrodynamic fine-structure energy levels of a muonic atom are perturbed by the non-Coulombic hyperfine interactions between the nucleus and the muon. Here we consider only the most important among these interactions: the magnetic dipole interaction associated with the nuclear spin, and the electric quadrupole interaction which is due to the possibly nonspherical distribution of charge within the nucleus. The finite nuclear radius is taken into account when computing the magnetic dipole interaction term.

The main correction to the hyperfine interactions which we do not consider here arises from the change in the nuclear potential due to vacuum polarization. A previous calculation ${ }^{4}$ of the hyperfine levels of $\mu^{-}{ }^{3} \mathrm{He}$, which included changes to the hyperfine interaction due to vacuum polarization and other small effects, agrees with our results to the desired level of accuracy. Furthermore, since we are considering nuclei which cannot be treated as spin- $\frac{1}{2}$ particles, the analysis of Ref. 4 cannot be directly applied to our calculations.

The hyperfine interactions split the quantumelectrodynamic fine-structure level $n L_{j}$ of a muonic atom with nuclear spin $I$ into the hyperfine-structure levels $n(2 F+1) L_{j}$ where $F$, the total angular momentum, satisfies

$$
|I-j| \leq F \leq I+j .
$$

The energy of the hyperfine-structure level $n(2 F+1) L_{j}$ is 
related to the energy of the corresponding fine-structure level $n L_{j}$ by

$$
\begin{aligned}
E_{\mathrm{hfs}}\left(n(2 F+1) L_{j}\right)= & E_{\mathrm{fs}}\left(n L_{j}\right)+E_{\mathrm{md}}\left(n(2 F+1) L_{j}\right) \\
& +E_{\text {eq }}\left(n(2 F+1) L_{j}\right),
\end{aligned}
$$

where $E_{\mathrm{md}}$ and $E_{\mathrm{eq}}$ are the corrections due to magnetic dipole and electric quadrupole interactions, respectively, and the values of $E_{\mathrm{fs}}$ are taken from I. The Uehling potential (first-order vacuum polarization) and finite nuclear size are treated exactly in that work, and other significant quantum-electrodynamic effects are treated perturbatively. The effect of nuclear polarization, though less certain, is also included in I. A full discussion of the methods used in the calculation of $E_{\mathrm{fs}}$ is given in I.

The magnetic dipole interaction perturbs the finestructure energy level by

$$
\begin{aligned}
& E_{\mathrm{md}}\left(n(2 F+1) L_{j}\right) \\
& \quad=\left(a_{j} / 2\right)[F(F+1)-j(j+1)-I(I+1)]\left(1+\delta_{L, 0} \epsilon\right),
\end{aligned}
$$

where

$a_{j}=\left\{\begin{array}{l}4 \alpha^{4} Z^{3}\left(\mu_{I} / I\right)\left(\mu^{3} / m_{\mu} m_{p}\right) c^{2} / 3 n^{3} \text { for } L=0 \\ \frac{\alpha^{4} Z^{3}\left(\mu_{I} / I\right)\left(\mu^{3} / m_{\mu} m_{p}\right) c^{2}}{n^{3}(2 L+1) j(j+1)} \text { for } L \geq 1 .\end{array}\right.$

In the above $m_{\mu}$ is the mass of the muon, $m_{p}$ the mass of the proton, $\mu$ the reduced mass of the system, and $\mu_{I}$ is the nuclear magnetic moment measured in nuclear magnetons (i.e., units of $e \hbar / 2 m_{p}$ ). Values of the physical constants used are the same as in I. Nuclear data are given in Table I. Following Zemach ${ }^{13}$ the finite-size correction $\epsilon$ is given by

$$
\epsilon=-2 Z\left(R / a_{0}\right)\left(\mu / m_{e}\right) 36 \sqrt{5} / 35 \sqrt{3},
$$

where we have employed a uniformly charged spherical model of the nucleus with root-mean-square radius $R$, and $a_{0}$ is the (electronic) Bohr radius. In lowest order, which is all we require here, the finite size of the nucleus only affects the magnetic dipole interaction term for $s$ states. The values of the nuclear radius used in these calculations

TABLE I. Nuclear input data for the calculation of hyperfine structure corrections (Ref. 14).

\begin{tabular}{cllll}
\hline \hline Nucleus & $\mu / m_{e}$ & $I$ & $\mu_{I}(\mathrm{~nm})$ & $Q\left(10^{-28} \mathrm{~m}^{2}\right)$ \\
\hline${ }^{3} \mathrm{He}$ & 199.289 & $\frac{1}{2}$ & -2.1276 & \\
${ }^{6} \mathrm{Li}$ & 202.940 & 1 & +0.82203 & -0.0008 \\
${ }^{7} \mathrm{Li}$ & 203.478 & $\frac{3}{2}$ & +3.25636 & -0.04 \\
${ }^{9} \mathrm{Be}$ & 204.198 & $\frac{3}{2}$ & -1.17745 & +0.05 \\
${ }^{10} \mathrm{~B}$ & 204.4514 & 3 & +1.8006 & +0.085 \\
${ }^{11} \mathrm{~B}$ & 204.659 & $\frac{3}{2}$ & +2.6885 & +0.041 \\
\hline \hline
\end{tabular}

are given along with the calculated energy levels in Table II.

The electric quadrupole interaction only acts when $I \geq 1$ and $j \geq 1$. In such a case the corresponding perturbation of the level $n L_{j}$ is given by

TABLE II. Contributions to the hyperfine energy levels (in $\mathrm{meV}$ ). For each case, $R$ is the assumed rms nuclear radius and $\Gamma_{2 p}$ is the $2 p$ level width.

\begin{tabular}{lcccr}
\hline \hline Level & $\begin{array}{c}\text { Fine-structure } \\
\text { splitting }\end{array}$ & $\begin{array}{c}\text { Magnetic } \\
\text { dipole } \\
\text { shift }\end{array}$ & $\begin{array}{c}\text { Electric } \\
\text { quadrupole } \\
\text { shift }\end{array}$ & Total \\
\hline & ${ }^{6} \mathrm{Li}(R=2.56$ & $\mathrm{fm}, \Gamma_{2 p}$ & $6.78 \mathrm{meV})$ & \\
$2 s_{1 / 2}$ & 0.0 & -108.8 & 0.0 & -108.8 \\
$4 s_{1 / 2}$ & 0.0 & 54.4 & 0.0 & 54.4 \\
$2 p_{1 / 2}$ & 1228.0 & -39.3 & 0.0 & 1188.7 \\
$4 p_{1 / 2}$ & 1228.0 & 19.7 & 0.0 & 1247.7 \\
$2 p_{3 / 2}$ & 1976.0 & -19.7 & -3.7 & 1952.7 \\
$4 p_{3 / 2}$ & 1976.0 & -7.9 & 2.9 & 1971.1 \\
$6 p_{3 / 2}$ & 1976.0 & 11.8 & -0.7 & 1987.1
\end{tabular}

${ }^{7} \mathrm{Li}\left(R=2.39 \mathrm{fm}, \Gamma_{2 p}=6.78 \mathrm{meV}\right)$

$\begin{array}{lrrrr}3 s_{1 / 2} & 0.0 & -363.9 & 0.0 & -363.9 \\ 5 s_{1 / 2} & 0.0 & 218.4 & 0.0 & 218.4 \\ 3 p_{1 / 2} & 1659.0 & -130.9 & 0.0 & 1528.1 \\ 5 p_{1 / 2} & 1659.0 & 78.5 & 0.0 & 1737.5 \\ 1 p_{3 / 2} & 2409.0 & -78.5 & -184.2 & 2146.3 \\ 3 p_{3 / 2} & 2409.0 & -57.6 & -36.8 & 2314.6 \\ 5 p_{3 / 2} & 2409.0 & -15.7 & 110.5 & 2503.8 \\ 7 p_{3 / 2} & 2409.0 & 47.1 & -36.8 & 2419.3\end{array}$

${ }^{9} \mathrm{Be}\left(R=2.52 \mathrm{fm}, \Gamma_{2 p}=21.5 \mathrm{meV}\right)$

$\begin{array}{lrrrr}3 s_{1 / 2} & 0.0 & 305.0 & 0.0 & 305.0 \\ 5 s_{1 / 2} & 0.0 & -183.0 & 0.0 & -183.0 \\ 3 p_{1 / 2} & -1302.0 & 113.4 & 0.0 & -1188.6 \\ 5 p_{1 / 2} & -1302.0 & -68.0 & 0.0 & -1370.0 \\ 1 p_{3 / 2} & 1077.0 & 68.0 & 551.6 & 1696.6 \\ 3 p_{3 / 2} & 1077.0 & 49.9 & 110.3 & 1237.2 \\ 5 p_{3 / 2} & 1077.0 & 13.6 & -331.0 & 759.7 \\ 7 p_{3 / 2} & 1077.0 & -40.8 & 110.3 & 1146.5\end{array}$

${ }^{10} \mathrm{~B}\left(R=2.45 \mathrm{fm}, \Gamma_{2 p}=52.7 \mathrm{meV}\right)$

$\begin{array}{rrrrr}6 s_{1 / 2} & 0.0 & -713.3 & 0.0 & -713.3 \\ 8 s_{1 / 2} & 0.0 & 535.0 & 0.0 & 535.0 \\ 6 p_{1 / 2} & -8902.0 & -271.9 & 0.0 & -9173.9 \\ 8 p_{1 / 2} & -8902.0 & 204.0 & 0.0 & -8698.1 \\ 4 p_{3 / 2} & -3074.0 & -163.2 & 882.4 & -2354.8 \\ 6 p_{3 / 2} & -3074.0 & -95.2 & -220.6 & -3389.8 \\ 8 p_{3 / 2} & -3074.0 & 0.0 & -735.3 & -3809.3 \\ 10 p_{3 / 2} & -3074.0 & 122.4 & 367.7 & -2584.0\end{array}$

\begin{tabular}{lrrrr} 
& $\mathrm{B}\left(R=2.42 \mathrm{rm}, \Gamma_{2 p}=52.7 \mathrm{meV}\right)$ & \\
$3 s_{1 / 2}$ & 0.0 & -1337.5 & 0.0 & -1337.5 \\
$5 s_{1 / 2}$ & 0.0 & 802.5 & 0.0 & 802.5 \\
$3 p_{1 / 2}$ & -8372.0 & -509.1 & 0.0 & -8881.1 \\
$5 p_{1 / 2}$ & -8372.0 & 305.5 & 0.0 & -8066.6 \\
$1 p_{3 / 2}$ & -2537.0 & -305.5 & 889.4 & -1953.0 \\
$3 p_{3 / 2}$ & -2537.0 & -224.0 & 177.9 & -2583.1 \\
$5 p_{3 / 2}$ & -2537.0 & -61.1 & -533.7 & -3131.7 \\
$7 p_{3 / 2}$ & -2537.0 & 183.3 & 177.9 & -2175.9 \\
\hline
\end{tabular}

a Measured relative to the $2 s_{1 / 2}$ fine-structure level. 


$$
\begin{aligned}
E_{\mathrm{eq}}\left(n(2 F+1) L_{j}\right) & \\
& =Q q_{j} \frac{3 K(K+1)-4 j(j+1)[I(I+1)]}{8 j(2 j-1) I(2 I-1)},
\end{aligned}
$$

where

$$
K=F(F+1)-I(I+1)-j(j+1),
$$

and $Q$ is the nuclear quadrupole moment measured in $\mathrm{m}^{2}$, the values for which are given in Table I. For a single bound muon

$$
q_{j}=\frac{\alpha^{4} Z^{3}\left(m_{e} c^{2} / \lambda\right)^{2}\left(\mu / m_{e}\right)^{3}(2 j-1)}{n^{3}(2 L+1) L(L+1)(j+1)},
$$

where $\hbar=\hbar / m_{e} c$ is the electronic Compton wavelength.

\section{RESULTS AND DISCUSSION}

The hyperfine energy levels are given in Table II, along with the contributions from the magnetic dipole and electric quadrupole interactions. Also listed are the $2 p$ level widths given in lowest order by

$$
\Gamma(2 p)=4.124 \times 10^{-5}\left(\mu / m_{e}\right) Z^{4} \mathrm{meV}
$$

for the $2 p \rightarrow 1 s$ electric dipole transition. The transition wavelengths are given in Table III. The results for ${ }^{3} \mathrm{He}$ agree with those of Borie ${ }^{4}$ to within the level width of the $2 p$ state. The results for $2 s_{1 / 2}-2 p_{1 / 2}$ transitions of ${ }^{6} \mathrm{Li}$, ${ }^{7} \mathrm{Li}$, and ${ }^{9} \mathrm{Be}$ differ by as much as $5 \%$ from the less accurate estimates of Feinberg and Chen. ${ }^{6}$ The present values are sufficiently precise for an accurate determination of the nuclear radius from observed transition frequencies.

For all cases but ${ }^{11} \mathrm{~B}$, the effect of increasing the assumed nuclear radius is to shift all the hyperfine levels up by about the same amount so that the ordering of these levels is not affected by the uncertainty in the nuclear radius. This is of particular importance in the case of the ${ }^{7} \mathrm{Li}$ hyperfine levels, where there are good prospects that an experimental measurement of the transition frequencies will lead to a precise determination of the nuclear radius. The case of ${ }^{11} \mathrm{~B}$ is exceptional in that the theoretical energies for the $3 s_{1 / 2}$ and $1 p_{3 / 2}$ levels cross as the radius varies from 2.32 to $2.52 \mathrm{fm}$. Several other transitions show dramatic variations of the predicted wavelength with $R$ as levels approach one another. The very-longwavelength transitions become essentially unobservable because of the large level widths shown in Table II. However, close approaches (if they exist) would enhance the parity violating effects discussed by Feinberg and Chen ${ }^{6}$ in the radiative decay of muonic states.

As pointed out in $\mathrm{I},{ }^{6} \mathrm{Li}$ and ${ }^{7} \mathrm{Li}$ are the best candidates for a measurement of the optical transition frequencies. At $5000 \AA$, the line width (from Table II) is about $15 \AA$ so that the hyperfine transitions are well resolved, and the nuclear radius uncertainty is sufficiently small that one knows where in the spectrum to look for the transitions. For the cases of ${ }^{9} \mathrm{Be},{ }^{10} \mathrm{~B}$, and ${ }^{11} \mathrm{~B}$, it would be necessary

\begin{tabular}{|c|c|c|c|}
\hline \multirow{2}{*}{ Transition } & \multicolumn{3}{|c|}{ Wavelength $(\AA)$} \\
\hline & $R=2.46 \mathrm{fm}$ & $R=2.56 \mathrm{fm}$ & $R=2.66 \mathrm{fm}$ \\
\hline $2 s_{1 / 2}-2 p_{1 / 2}$ & 7972.8 & 9556.2 & 12031.8 \\
\hline $2 s_{1 / 2}-4 p_{1 / 2}$ & 7681.4 & 9140.5 & 11380.2 \\
\hline $4 s_{1 / 2}-2 p_{1 / 2}$ & 8910.9 & 10930.9 & 14286.6 \\
\hline $4 s_{1 / 2}-4 p_{1 / 2}$ & 8548.4 & 10390.4 & 13377.0 \\
\hline $2 s_{1 / 2}-2 p_{3 / 2}$ & 5349.9 & 6014.5 & 6909.6 \\
\hline $2 s_{1 / 2}-4 p_{3 / 2}$ & 5307.8 & 5961.3 & 6839.6 \\
\hline $4 s_{1 / 2}-2 p_{3 / 2}$ & 5756.6 & 6531.5 & 7598.3 \\
\hline $4 s_{1 / 2}-4 p_{3 / 2}$ & 5707.9 & 6468.8 & 7513.6 \\
\hline $4 s_{1 / 2}-6 p_{3 / 2}$ & 5666.1 & 6415.2 & 7441.4 \\
\hline${ }^{7} \mathrm{Li}$ & $R=2.29 \mathrm{fm}$ & $R=2.39 \mathrm{fm}$ & $R=2.49 \mathrm{fm}$ \\
\hline $3 s_{1 / 2}-3 p_{1 / 2}$ & 5804.2 & 6553.0 & 7568.7 \\
\hline $3 s_{1 / 2}-5 p_{1 / 2}$ & 5285.9 & 5900.0 & 6710.8 \\
\hline $5 s_{1 / 2}-3 p_{1 / 2}$ & 7989.2 & 9466.5 & 11721.5 \\
\hline $5 s_{1 / 2}-5 p_{1 / 2}$ & 7039.2 & 8161.4 & 9784.3 \\
\hline $3 s_{1 / 2}-1 p_{3 / 2}$ & 4501.4 & 4939.3 & 5494.8 \\
\hline $3 s_{1 / 2}-3 p_{3 / 2}$ & 4242.2 & 4628.9 & 5113.5 \\
\hline $3 s_{1 / 2}-5 p_{3 / 2}$ & 3984.2 & 4323.5 & 4743.3 \\
\hline $5 s_{1 / 2}-3 p_{3 / 2}$ & 5302.0 & 5914.8 & 6722.6 \\
\hline $5 s_{1 / 2}-5 p_{3 / 2}$ & 4905.0 & 5425.0 & 6097.0 \\
\hline $5 s_{1 / 2}-7 p_{3 / 2}$ & 5074.7 & 5633.4 & 6361.4 \\
\hline${ }^{9} \mathrm{Be}$ & $R=2.42 \mathrm{fm}$ & $R=2.52 \mathrm{fm}$ & $R=2.62 \mathrm{fm}$ \\
\hline $3 s_{1 / 2}-3 p_{1 / 2}$ & 17734.4 & 8301.0 & 5347.6 \\
\hline $3 s_{1 / 2}-5 p_{1 / 2}$ & 14080.6 & 7401.9 & 4959.5 \\
\hline $5 s_{1 / 2}-3 p_{1 / 2}$ & 59361.0 & 12329.5 & 6765.1 \\
\hline $5 s_{1 / 2}-5 p_{1 / 2}$ & 31768.2 & 10445.1 & 6155.7 \\
\hline $3 s_{1 / 2}-1 p_{3 / 2}$ & 5666.9 & 8909.6 & 22010.6 \\
\hline $3 s_{1 / 2}-3 p_{3 / 2}$ & 7173.1 & 13300.5 & 119349.3 \\
\hline $3 s_{1 / 2}-5 p_{3 / 2}$ & 9911.4 & 27271.7 & 33180.3 \\
\hline $5 s_{1 / 2}-3 p_{3 / 2}$ & 5588.1 & 8730.1 & 21025.9 \\
\hline $5 s_{1 / 2}-5 p_{3 / 2}$ & 7120.7 & 13152.8 & 110579.3 \\
\hline $5 s_{1 / 2}-7 p_{3 / 2}$ & 5826.3 & 9325.7 & 24848.2 \\
\hline${ }^{10} \mathrm{~B}$ & $R=2.35 \mathrm{fm}$ & $R=2.45 \mathrm{fm}$ & $R=2.55 \mathrm{fm}$ \\
\hline $6 s_{1 / 2}-6 p_{1 / 2}$ & 1878.4 & 1465.4 & 1193.6 \\
\hline $6 s_{1 / 2}-8 p_{1 / 2}$ & 2024.4 & 1552.8 & 1251.0 \\
\hline $8 s_{1 / 2}-6 p_{1 / 2}$ & 1578.2 & 1277.0 & 1066.3 \\
\hline $8 s_{1 / 2}-8 p_{1 / 2}$ & 1680.0 & 1342.9 & 1111.8 \\
\hline $6 s_{1 / 2}-4 p_{3 / 2}$ & 56519.3 & 7553.1 & 3474.3 \\
\hline $6 s_{1 / 2}-6 p_{3 / 2}$ & 15201.4 & 4632.4 & 2693.2 \\
\hline $6 s_{1 / 2}-8 p_{3 / 2}$ & 10038.0 & 4004.7 & 2468.3 \\
\hline $8 s_{1 / 2}-6 p_{3 / 2}$ & 5986.3 & 3159.1 & 2121.4 \\
\hline $8 s_{1 / 2}-8 p_{3 / 2}$ & 4978.0 & 2854.0 & 1979.3 \\
\hline $8 s_{1 / 2}-10 p_{3 / 2}$ & 9798.5 & 3975.3 & 2460.6 \\
\hline${ }^{11} \mathrm{~B}$ & $R=2.32 \mathrm{fm}$ & $R=2.42 \mathrm{fm}$ & $R=2.52 \mathrm{fn}$ \\
\hline $3 s_{1 / 2}-3 p_{1 / 2}$ & 2177.2 & 1643.6 & 1310.7 \\
\hline $3 s_{1 / 2}-5 p_{1 / 2}$ & 2540.6 & 1842.5 & 1434.2 \\
\hline $5 s_{1 / 2}-3 p_{1 / 2}$ & 1580.0 & 1280.4 & 1070.1 \\
\hline $5 s_{1 / 2}-5 p_{1 / 2}$ & 1763.0 & 1398.0 & 1151.0 \\
\hline $3 s_{1 / 2}-1 p_{3 / 2}$ & 10047.1 & 20140.9 & 4896.5 \\
\hline $3 s_{1 / 2}-3 p_{3 / 2}$ & 20528.2 & 9953.4 & 3920.9 \\
\hline $3 s_{1 / 2}-5 p_{3 / 2}$ & 223961.8 & 6910.1 & 3341.2 \\
\hline $5 s_{1 / 2}-3 p_{3 / 2}$ & 8006.9 & 3662.2 & 2344.0 \\
\hline $5 s_{1 / 2}-5 p_{3 / 2}$ & 5912.2 & 3151.5 & 2123.7 \\
\hline $5 s_{1 / 2}-7 p_{3 / 2}$ & 10864.3 & 4163.0 & 2539.5 \\
\hline
\end{tabular}
first to locate one of the less sensitive short-wavelength transitions.
TABLE III. Calculated transition wavelengths for different assumed values of the rms nuclear radius $R$. 


\section{ACKNOWLEDGMENT}

Research support by the Natural Sciences and Engineering Research Council of Canada is gratefully acknowledged.

${ }^{1}$ A. Bertin, G. Carboni, J. Duclos, U. Grastaldi, G. Gorini, G. Neri, J. Picard, O. Pitzurra, A. Placci, E. Polacco, G. Torelli, A. Vitale, and E. Zavattini, Phys. Lett. B 55, 411 (1975).

${ }^{2}$ G. Carboni, G. Gorini, L. Palffy, F. Palmonari, and E. Zavattini, Nucl. Phys. A 278, 381 (1977); G. Carboni, G. Gorini, E. Iacopini, L. Palffy, F. Palmonari, G. Torelli, and E. Zavattini, Phys. Lett. B 73, 229 (1978).

${ }^{3}$ For a review, see E. Borie and G. A. Rinker, Rev. Mod. Phys. 54, 67 (1982).

${ }^{4}$ E. Borie, Z. Phys. A 297, 17 (1980).

${ }^{5}$ G. W. F. Drake and L. L. Byer, Phys. Rev. A 32, 713 (1985).

${ }^{6}$ G. Feinberg and M. Y. Chen, Phys. Rev. D 10, 190 (1974); 10, 3789 (1974).

${ }^{7}$ A. N. Moskalev, Pis'ma Zh. Eksp. Teor. Fiz. 19, 394 (1974)
[JETP Lett. 19, 216 (1974)].

${ }^{8}$ H. P. von Arb, F. Dittus, H. Heeb, H. Hoffer, F. Kottman, S. Niggli, R. Schaeren, D. Taggu, J. Unternahrer, and P. Egelhof, Phys. Lett. B 136, 232 (1984). See also, M. Eckhause, P. Guss, D. Joyce, J. R. Kane, R. T. Siegel, W. Vulcan, R. E. Welsh, and R. Whyley, Phys. Rev. A 33, 1743 (1986), for recent measurements at high pressure.

${ }^{9}$ R. O. Mueller, V. W. Hughes, H. Rosenthal, and C. S. Wu, Phys. Rev. A 11, 1175 (1975).

10J. S. Cohen and J. N. Bardsley, Phys. Rev. A 23, 46 (1981).

${ }^{11}$ G. Carboni and O. Pitzurra, Nuovo Cimento B 25, 367 (1975).

12J. S. Cohen, Phys. Rev. A 25, 1791 (1982).

${ }^{13}$ A. C. Zemach, Phys. Rev. 104, 1771 (1956).

${ }^{14}$ G. H. Fuller, J. Phys. Chem. Ref. Data 5, 835 (1976). 